\title{
Development of a Reflectance Photo Plethysmograph for the Estimation of Pulse Transit Time
}

\author{
Yatavakilla Amarendra Nath, Vuyyuru Rachana, Aryasomayajula Sri Teja, Talasila Srujan Kumar, \\ Alaka Chandan
}

\begin{abstract}
Pulse transit time is considered as a vital physiological parameter for assessing several cardiac disorders. It was proven that pulse transit time provides accurate diagnosis in estimation of blood pressure. In this paper, we demonstrate an instrumented hardware for the development of reflectance photoplethysmograph. The designed system uses two reflectance sensors at two different arterial locations for the estimation of pulse transit Time. Developed hardware is used for the calculation or estimation of the pulse transit time from the two photoplethysmograph signals obtained, one from the wrist and the other from the finger respectively, estimated pulse transit time is compared against the gold standard equipment, the results indicate the estimated values are within the limits of medical diagnostic values.
\end{abstract}

Index Terms-Photoplethysmograph, Pulse transit time, Sensors, Reflectance.

\section{INTRODUCTION}

The number of persons suffering from cardiovascular diseases is on a rise in this stressful lifestyle. Patients are being monitored such that a potential serious and lifethreatening situation can be detected in time of emergency for medical doctors to act. Diagnoses of such ailments are often complicated due to rapid and dynamic variation in the associated parameters of the individual under examination. Patients suffering from, for example heart diseases need to be continuously monitored for their cardiovascular system i.e. heart and blood function.

Revised Manuscript Received on December 15, 2019

Yatavakilla Amarendra Nath, School of Biomedical Engineering, Department of Electronics and Communication Engineering, Vignan's Foundation for Science, Technology \& Research, Vadlamudi, Guntur, Andhra Pradesh-522213, India.

e-mail:amar.yatavakilla@gmail.com

Vuyyuru Rachana, School of Biomedical Engineering, Department of Electronics and Communication Engineering, Vignan's Foundation for Science, Technology \& Research, Vadlamudi, Guntur, Andhra Pradesh522213 , India

Aryasomayajula Sri Teja, School of Biomedical Engineering, Department of Electronics and Communication Engineering, Vignan's Foundation for Science, Technology \& Research, Vadlamudi, Guntur, Andhra Pradesh-522213, India

Talasila Srujan Kumar, School of Biomedical Engineering, Department of Electronics and Communication Engineering, Vignan's Foundation for Science, Technology \& Research, Vadlamudi, Guntur, Andhra Pradesh-522213, India

Alaka Chandan, School of Biomedical Engineering, Department of Electronics and Communication Engineering, Vignan's Foundation for Science, Technology \& Research, Vadlamudi, Guntur, Andhra Pradesh522213, India
To enhance the life quality of these patients, monitoring units should be as mobile, discreet, and autonomic as possible such that patients will not feel a burden by being attached to units making a regular daily life impossible. They should also feature wearable and wireless communication for transmission of data for evaluation by a professional and to send out emergency signals. Mobile and autonomic solutions would be of great benefit for people involved in traumatic accidents and for battlefield soldiers.

In this case rescuers attach monitoring units to the wounded patients such that their condition can be followed during transport to hospital or by doctors ready to receive the patient at the hospital. Monitoring fire fighters, combat soldiers, rescues workers, or just professionals performing tasks that are physically exhausting could help saving lives by warning the professional or the supervisor such that action can be taken before a collapse occurs, hence there is a very robust and demanding situation for the development of wearable medical equipment[12]. Medically, it is important to monitor change in the blood volume, which is estimated using PPG in which light of suitable wavelength (between $600 \mathrm{~nm}$ to $1300 \mathrm{~nm}$ ) is passed through a body part and the resultant scattered or transmitted light is measured with the help of a photo detector. The detected light is directly proportional to the pulsating volume change in the blood [8]. During each cardiac cycle the heart pumps blood to the periphery. Due to pulsatile pumping of heart, the blood volume at any part of body during a period of time varies. These variations can be detected using PPG. PPG can be used to monitor the heart rate, cardiac cycle, breathing, hypovolemia. The implementation of this work helps to overcome the complexity of distance measurement and also reduces the use of ECG for the measurement of Pulse transit time. A unique sensor architecture is employed to estimate pulse wave velocity (PWV), this method is applied to calibrate peripheral pulse transit time measurements to arterial blood pressure. This prototype assumes laminar blood flow from the heart chamber to the fingertip through a rigid pipe, the artery $[1,6]$ and estimates the difference in pressure between the two sites from the same artery. It was suggested that pulse wave velocity can be estimated using pulse transit time measured from two different arterial locations [1].

Many wearable mobile monitoring systems are available today such as Bionomadix and oximeter PPG sensor shown in Fig 1.1 and fig 1.2. 
For patients who need monitoring without affecting their normal life, such systems are required. For traumatic patients, rescuers need not have to attach all the sensors and carry the boxes along with the patient. A small autonomous system would make the rescuers' work simpler, and they could focus on the patient.
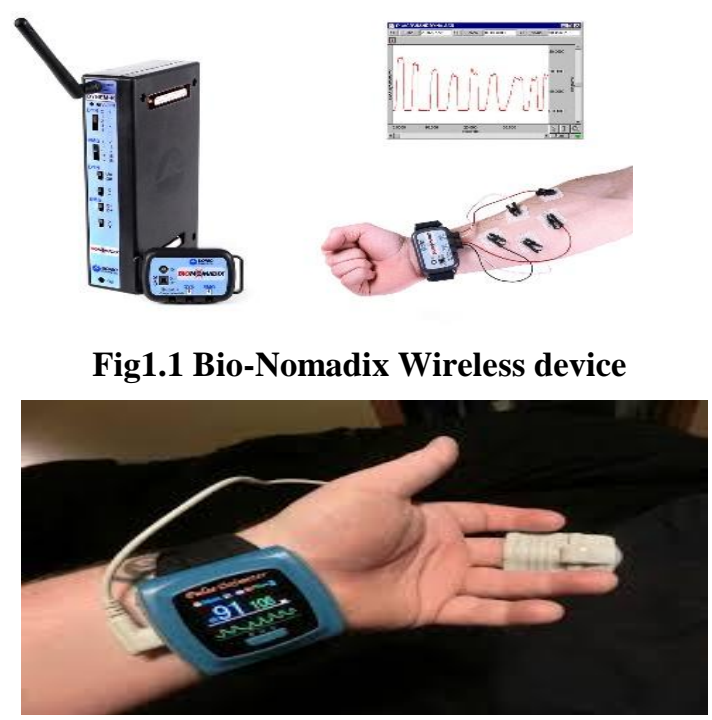

Fig. 1.2 Wearable Pulse Oximeter

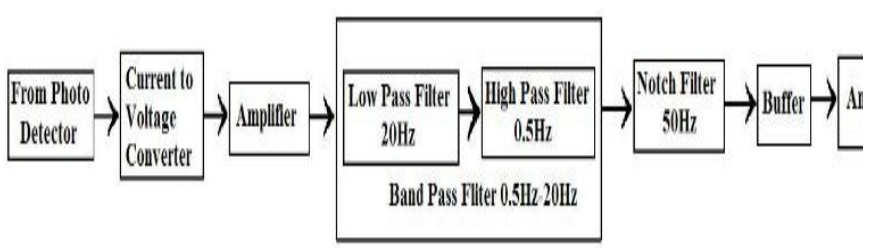

Fig. 2.1 PPG Block diagram

\section{MATERIALS AND METHODS}

The designed PPG circuits were used to acquire signals from different body locations, in this work we have used the primary location as wrist and finger, and we made sure that the diagnostic information of the PPG is not lost by enclosing the sensors in a black tape. These circuits are interfaced to the computer using a DAQ PCI-6104 and PPG data is acquired in the system. These data are further used in the MATLAB program to calculate the PTT. The pulse transit time obtained is compared with a standard BIOPAC device.

\section{A. Hardware Design}

The figure 2.1 illustrates the block diagram of the developed PPG system, the hardware consists of a low pass Butterworth filter and a high pass Butterworth filter $\left(2^{\text {nd }}\right.$ order) with lower cutoff frequency of $20 \mathrm{~Hz}$ and higher cutoff frequency of $0.5 \mathrm{~Hz}$ respectively. The signal is preamplified with a gain of 1000 . Further processing of signal is performed by converting analog signal to digital signal with a notch filter being implemented in the final stage to remove $50 \mathrm{~Hz}$ power line noise[11].

\section{B. Design Criteria}

In this work lot of care is taken to reduce the discomfort of the wearer, the sensor must be thin and unobtrusive. If possible, the sensor can be made in decorative manner such that it is not obvious to the others. Besides that, the sensor
Fig1.1 Bio-Nomadix Wireless device

should not be obtrusive to the wearer's daily activities. In most cases, noise and signal would be mixed up during acquisition, this was eliminated by attaching the sensor rigidly to the finger, ulnar artery and digital artery were selected for unobtrusive data acquisition. The shielding of the sensor is difficult due to the sensitivity of the sensor to light. To reduce the noise caused by the ambient light, photodiode has to be optically shielded from it. We have used a rigid flexible black tape to protect the sensors from unwanted light which in turn helps in avoiding unwanted voltage[5,8].

\section{Circuit Design}

Photo Transmitter:

Red LED ELM4000 manufactured by measurement specialists with current and voltage rating of $20 \mathrm{~mA}$ and $2.3 \mathrm{~V}$ respectively. IR LED EL23G manufactured by Kodenshi with current rating and voltage rating of $60 \mathrm{~mA}$ and $1.6 \mathrm{~V}$ respectively.

\section{Photo Detector:}

EPM4001 manufactured by measurement specialists and HP307R2 manufactured by Kodenshi are used to detect Red light and IR light respectively. OPA-07 is used as an OPAMP which is operated with dual power supply $+5 \mathrm{~V}$ to $-5 \mathrm{~V}$.

\section{DAQ interface (tool used)}

Measurement Computing PCI-6014 DAQ is a 16Channel, 16-bit, $200 \mathrm{kS} / \mathrm{s}$ DAQ Board with 8 Digital I/O and Two 16-Bit Analog Outputs which is used to interface the PPG signals to the computer along with TracerDAQ software included for acquiring and displaying data and generating signals. The DAQ module samples the signal at the rate of 100sample/sec.

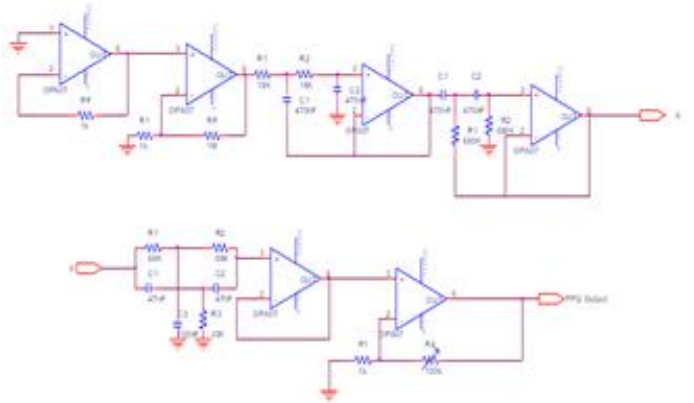

Fig.2.2 Total PPG Circuit

\section{RESULTS}

It compares the resultant PTT values obtained from $\mathrm{R}$ peak derived ECG- finger PPG with respect to wrist PPG - finger PPG. It also shows the results obtained from the hardware developed and the software code written in MATLAB which is implemented on the PPG signals obtained from the developed hardware[4].

\section{A. Hardware development}

The hardware developed for the Photoplethysmograph is put together on the breadboard and then tested and transformed to PCB for the essential parameters data collection and the results are as follows: shed By:

Blue Eyes Intelligence Engineering

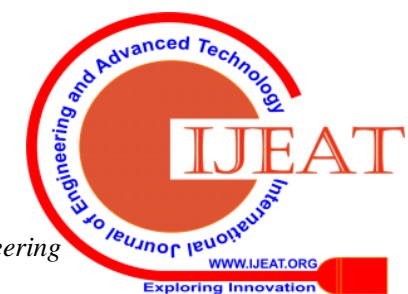




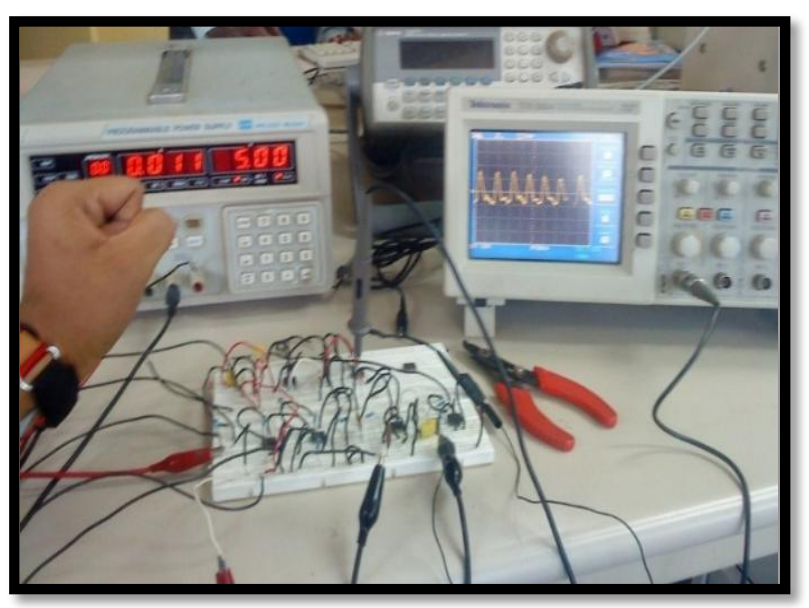

Fig.3.1 Shows the reflectance PPG signal obtained from the wrist which is obtained from the instrumented hardware developed on breadboard is displayed on the CRO

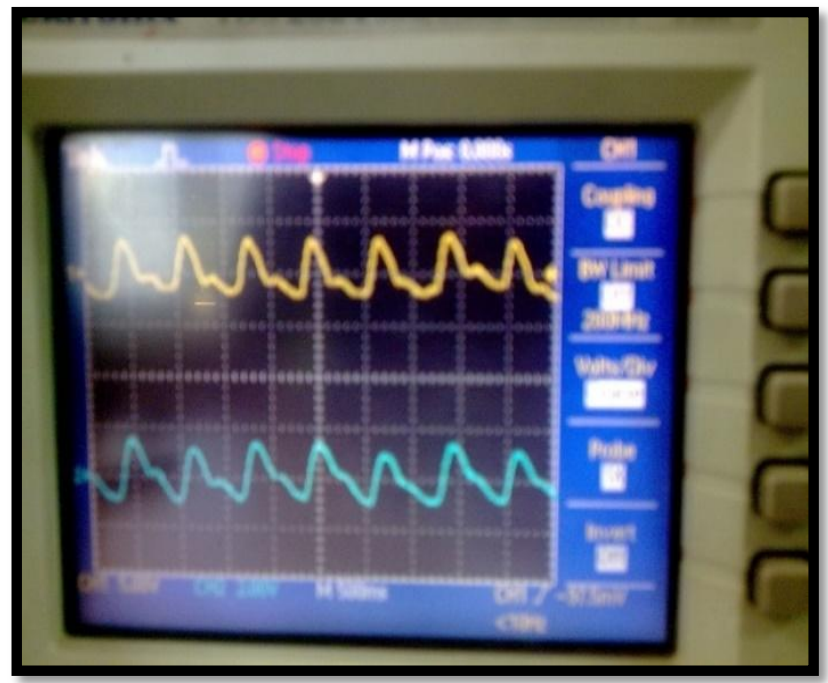

Fig.3.2 Shows the Photoplethysmogram signals obtained from ulnar (yellow) and digital (blue) artery respectively.

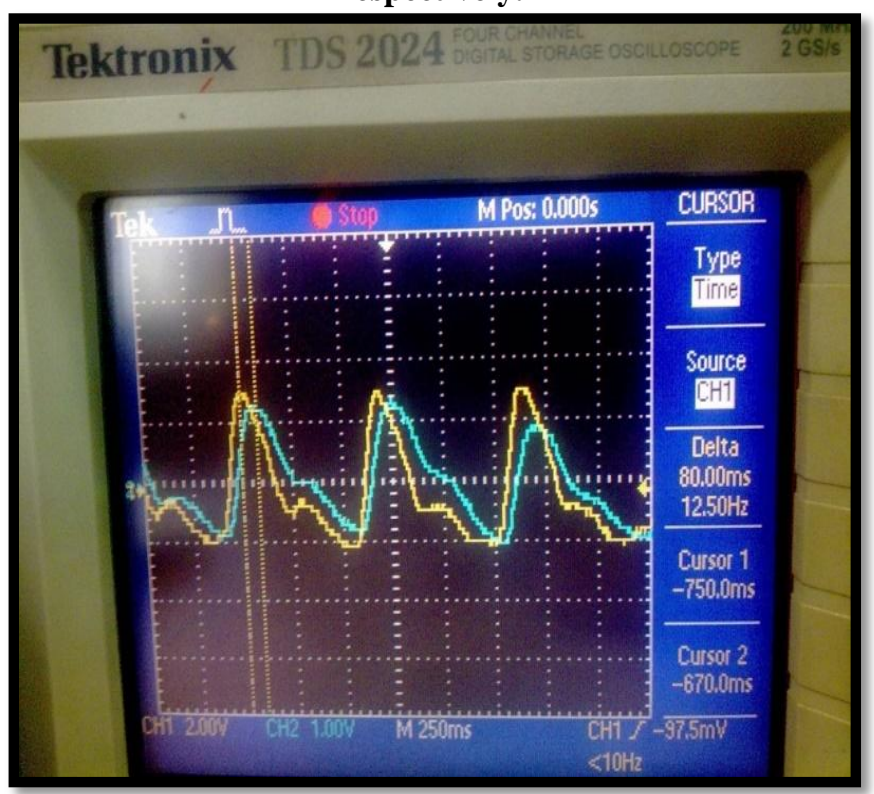

Fig.3.3 shows the overlapping of ulnar(wrist) and digital

(finger) Photoplethysmogram, which shows the time difference value measured as $80 \mathrm{~ms}$.

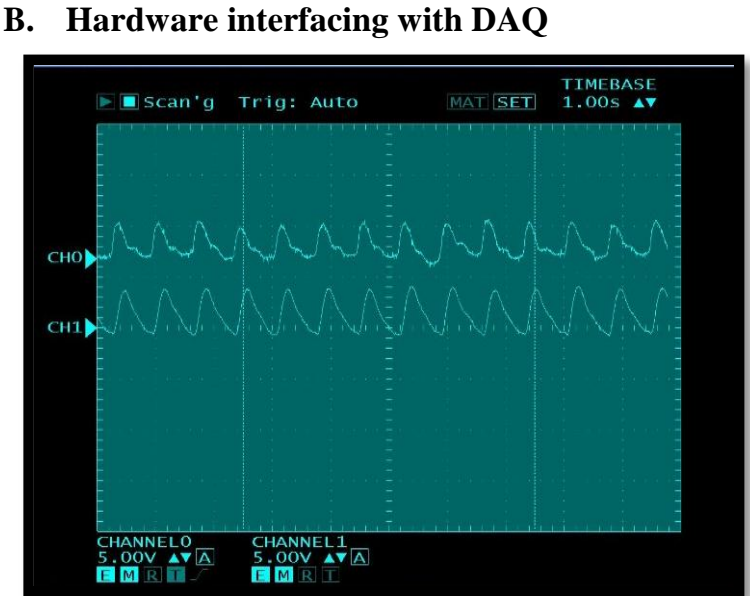

Fig.3.4: Snapshot of acquiring PPG signals using Daq and the software TraceDAQ

\section{PTT calculation using MATLAB}

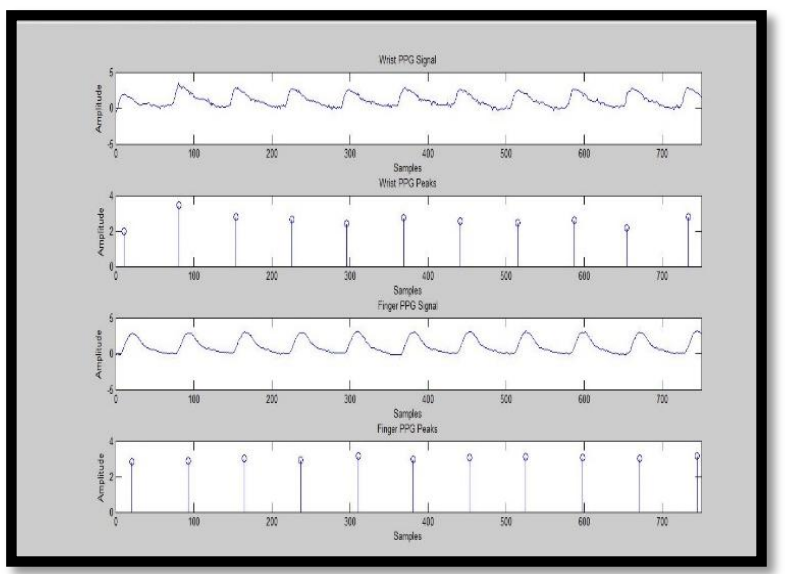

Fig.3.5 Represents the peaks of different PPG signals filtered

\begin{tabular}{|c|c|c|c|}
\hline Name & $\begin{array}{c}\text { Pulse } \\
\text { tansit } \\
\text { time } \\
\text { (Wrist- } \\
\text { Finger) } \\
\text { ms }\end{array}$ & $\begin{array}{c}\text { Dst(Wrist- } \\
\text { Finger) } \\
\mathrm{cm}\end{array}$ & $\begin{array}{c}\text { Pulse wave } \\
\text { Velocity(Wrist- } \\
\text { Finger) } \\
\mathrm{m} / \mathrm{s}\end{array}$ \\
\hline Subject1 & 80 & 20 & 2.5 \\
\hline Subject2 & 110 & 22 & 2.0 \\
\hline Subject3 & 70 & 18 & 02.571 \\
\hline Subject3 & 90 & 23 & 02.555 \\
\hline Subject4 & 80 & 18 & 02.25 \\
\hline
\end{tabular}

Table 1 The table depicts the particulars collected from the different subjects for the estimation of Pulse wave velocity

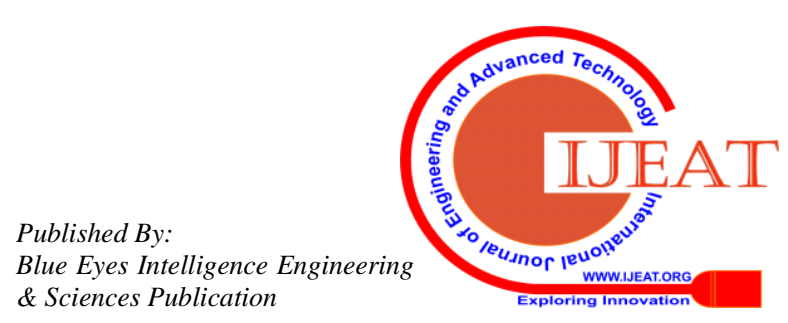




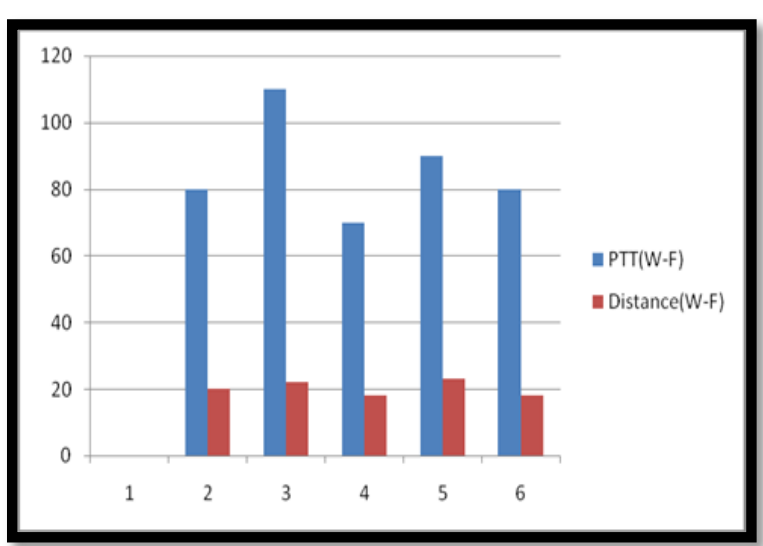

Fig.3.6 Graph plot of Distance from WRISTFINGER and PTT obtained from Mat lab algorithm

These are the results obtained from the designed PPG wrist and PPG finger circuits interfaced with DAQ and by calculating PTT using MATLAB.

D. PTT calculation by interfacing circuit with BIOPAC

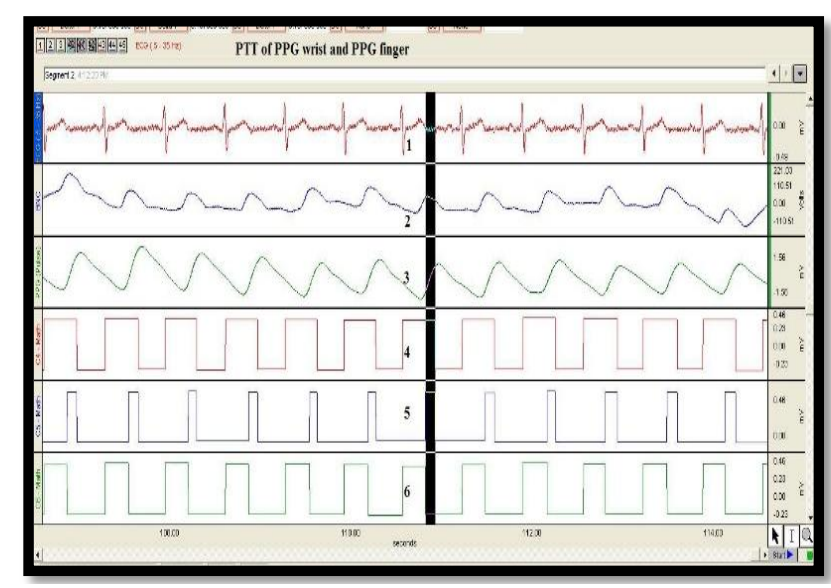

Fig.3.7 Snapshot of BIOPAC showing PTT of PPG wrist and PPG finger with result 107 milliseconds

Table.2. Details of particulars obtained from 5 different subjects

\begin{tabular}{|c|c|c|c|c|c|c|}
\hline Name & $\begin{array}{l}\text { PT } \\
\text { T } \\
\text { (E- } \\
\text { F) } \\
\text { ms }\end{array}$ & $\begin{array}{l}\text { PT } \\
\text { T } \\
(\text { E- } \\
\text { W) } \\
\text { ms }\end{array}$ & $\begin{array}{l}\text { PT } \\
\text { T } \\
\text { (W } \\
-\mathrm{F}) \\
\mathrm{ms}\end{array}$ & $\begin{array}{l}\text { PW } \\
\text { V } \\
(\mathrm{E}- \\
\mathrm{F}) \\
\mathrm{m} / \mathrm{s}\end{array}$ & $\begin{array}{l}\mathrm{PW} \\
\mathrm{V} \\
(\mathrm{E}- \\
\mathrm{W}) \\
\mathrm{m} / \mathrm{s}\end{array}$ & $\begin{array}{l}\mathrm{PWV} \\
(\mathrm{W}- \\
\mathrm{F}) \\
\mathrm{m} / \mathrm{s}\end{array}$ \\
\hline $\begin{array}{l}\text { Subject } \\
1\end{array}$ & 330 & 250 & 80 & 2.57 & 2.6 & 2.5 \\
\hline $\begin{array}{l}\text { Subject } \\
2\end{array}$ & 360 & 270 & 90 & 2.5 & $\begin{array}{l}2.59 \\
2\end{array}$ & $\begin{array}{l}2.44 \\
4\end{array}$ \\
\hline $\begin{array}{l}\text { Subject } \\
3\end{array}$ & 300 & 230 & 70 & $\begin{array}{l}2.66 \\
6\end{array}$ & $\begin{array}{l}2.72 \\
7\end{array}$ & $\begin{array}{l}2.57 \\
1\end{array}$ \\
\hline $\begin{array}{l}\text { Subject } \\
4\end{array}$ & 350 & 260 & 90 & $\begin{array}{l}2.71 \\
4\end{array}$ & $\begin{array}{l}2.88 \\
4\end{array}$ & $\begin{array}{l}2.55 \\
5\end{array}$ \\
\hline $\begin{array}{l}\text { Subject } \\
5\end{array}$ & 300 & 230 & 70 & $\begin{array}{l}2.66 \\
6\end{array}$ & $\begin{array}{l}2.69 \\
6\end{array}$ & $\begin{array}{l}2.57 \\
1\end{array}$ \\
\hline
\end{tabular}

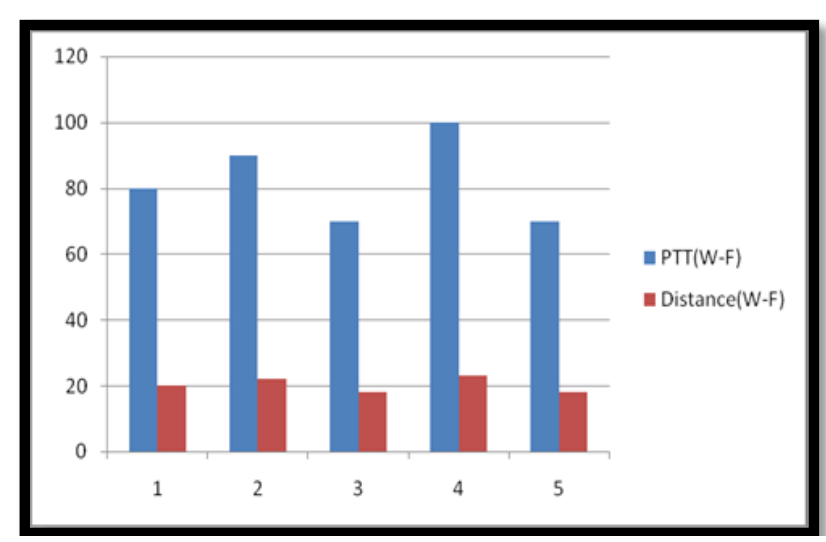

Fig.3.8 Graph shows the Pulse transit time variation with respect to ECG, wrist PPG and PPG finger

\section{CONCLUSION}

The outcome clearly show that PTT can be estimated using Photoplethysmograph circuits, one of them being the wrist and other finger circuit which replaces the utilization of Electrocardiograph and prevents the need for error corrections associated with hydrostatic pressure changes when hand movements were performed during daily activities[2]. The Hardware unit developed, and software algorithm implemented to calculate Pulse Transit Time is validated using the standardized BIOPAC instrument, the validation results were satisfactory for the estimation of PTT from wrist to finger. In future, in order to reduce the size and also to make the equipment wearable, we intend to implement hardware by integrating xigbee module for unobtrusive data transmission.motion induced pressure variation changes will be compensated with a Gyroscope and pressure sensor integration, one located in the sensor place on the digital artery housing and a second located at the ulnar artery. There will be variation in pressure with respect to height from heart which will show its effect in calculating pulse transit time so, in order to counter act, the implementation of adaptive hydrostatic pressure. This project will help in assessing the pulse wave velocity and pulse transit time estimation, which will be a valuable marker for pre-clinical assessment of cardio vascular health.

\section{Acknowledgement}

We are grateful to Vignan's Foundation for Science technology and Research University for providing financial support and research facility for this work

\section{REFERENCES}

1. Asada H, et al., "Mobile Monitoring with Wearable Photoplethysmogrphic Biosensors," IEEE Engineering in Medicine and Biology Magazine, 22(3), pp. 28-40, 2003.

2. Cheah Kim Wei, "Photoplethysmography Blood Pressure Measurement" Department of Mechanical Engineering, National University of Singapore.

3. Devin B. McCombie,et al; "Adaptive blood pressure estimation from wearable $P P G$ sensors using peripheral artery pulse wave velocity measurements and multi-channel blind identification of local arterial dynamics"28th IEEE EMBS Annual International Conference New York City, USA, Aug 30-Sept 3, 2006

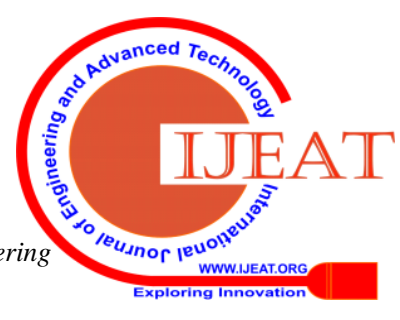


4. Fredrik Gustafsson and Niclas Bergman, "MATLAB for Engineer Explained", Published by Springer-Verlag London Limited.

5. K. Ashoka Reddy; "Novel Method for Performance Enhancement of Pulse Oximeters" PhD Thesis, Dept of Electrical Engineering, IIT Madras 2008.

6. Kejia Li "Wireless Reflectance Pulse Oximeter Design and Photoplethysmographic Signal Processing "B.S., Zhejiang University, 2008.

7. Lass J, Meigas K, Karai D, Kattai R, Kaik J, Rossmann M Continuous Blood Pressure Monitoring During Exercise Using Pulse Wave Transit Time Measurement. Proceedings of the $26^{\text {th }}$ Annual International Conference of the IEEE EMBS 2004; September: 2239-2242.

8. Michael Maguire and Tomas Ward; "The Photoplethysmograph as an instrument for physiological measurement" Department of Electronic Engineering, NUI Maynooth 2002

9. Ramakant A. Gayakwad, "OP AMP and Linear Integrated Circuits and their applications" $4^{\text {th }}$ Edition

10. Rasmus G. Haahr; "Reflectance Pulse Oximetry Sensor for the Electronic Patch" MSc Thesis, MIC - Department of Micro and Nanotechnology Technical University of Denmark, November 2006.

11. Rudrapratap, "MATLAB7 A Quick Introduction for Scientist and Engineers", Published by Oxford University Press, New Delhi.

12. Russell Dresher, "Wearable Forehead Pulse Oximetry: Minimization of Motion and Pressure Artifacts", MSc Thesis, Worcester Polytechnic Institute 